AIAA-99-0699

\title{
ULTRASOUND IMAGING SYSTEM IMPLEMENTATION AND IGNITION PROTOCOL FOR THE MICROGRAVITY SMOLDERING COMBUSTION (MSC) EXPERIMENTS
}

\author{
David C. Walther, Ralph A. Anthenien ${ }^{\dagger}$, Mark Roslon and A. Carlos Fernandez-Pello* \\ University of California, Berkeley \\ Berkeley, CA 94720 \\ and \\ David L. Urban \\ NASA Lewis Research Center \\ Cleveland, $\mathrm{OH} 44135$ \\ ${ }^{\dagger}$ Current address: Air Force Research Laboratory/ Propulsion Directorate \\ Wright-Patterson AFB, OH 45433 \\ *Author to whom correspondence should be directed
}

\begin{abstract}
The Microgravity Smoldering Combustion (MSC) experiment is a study of the smolder characteristics of porous combustible materials in a microgravity environment. The objective of the study is to provide a better understanding of the controlling mechanisms of smolder, both in microgravity and normal earth gravity. Experiments have been conducted aboard the NASA Space Shuttle in the Get Away Special Canister (GAS-CAN), an apparatus requiring completely remote operation. Future GAS-CAN experiments will utilize an ultrasound imaging system (UIS) which has been incorporated into the MSC experimental apparatus. Thermocouples are currently used to measure temperature and reaction front velocities. A less intrusive method is desirable, however, as smolder is a very weak reaction and it has been found that heat transfer along the thermocouple is sufficient to affect the smolder reaction. It is expected that the UIS system will eventually replace the existing array of thermocouples as a non-intrusive technique without compromising data acquisition. The UIS measures line of sight permeability, providing information about the reaction front position and extent.
\end{abstract}

Additionally, the ignition sequence of the MSC experiments has been optimized from previous experiments to provide longer periods of selfsupported smolder. An ignition protocol of a fixed power to the igniter for a fixed time is now implemented. This, rather than a controlled temperature profile ignition protocol at the igniter surface, along with the UIS system, will allow for better study of the effect of gravity on a smolder reaction.

\section{Introduction}

Smoldering is defined as a non-flaming, selfsustaining, propagating, exothermic, surface reaction, deriving its principal heat from heterogeneous oxidation of the fuel [1]. If the material is sufficiently permeable, smoldering is not confined to its outer surface and can propagate as a reaction wave through the interior of the material. Many materials can sustain smoldering, ranging from dry organic materials to polymeric foams. The ignition, propagation, transition to flaming and extinction of the smolder reaction are controlled by complex, thermo-chemical mechanisms that are not well understood. 
As a basic combustion problem, smoldering encompasses a number of fundamental processes including: heat and mass transfer in porous media; endothermic pyrolysis of the combustible; ignition, propagation and extinction of oxidative, heterogeneous, exothermic reactions at the gas/solid interface; and the onset of gas phase reactions (flaming). In the context of fire safety, smoldering is of concern because it can propagate slowly in the material interior and go undetected for long periods of time, typically yielding a substantially higher conversion of fuel to toxic compounds than does flaming (albeit more slowly), and may undergo a sudden transition to flaming [2]. Various fire studies since the early 1970's have shown that approximately $40 \%$ of United States fire deaths can be attributed to smoldering of household furniture [3]. Adding to the hazard are the facts that minimum smolder ignition energies are typically lower than for flaming combustion and smoldering can occur under lower oxygen concentrations than normal air. Smolder of cable insulation, another common fire hazard, is of particular concern in the space program; to date there have been a few minor incidents of overheated and charred cables and electrical components reported on Space Shuttle flights [4,5]. Recently, the planned establishment of the International Space Station and other space facilities has increased interest in the study of smoldering in microgravity because of the need to preempt the possibility, and/or to minimize the effect of a smolder initiated fire during the operation of these facilities [6].

There are two classifications for the onedimensional propagation of a smolder reaction: opposed and forward [1], defined according to the direction in which fuel and oxidizer enter the reaction zone. In opposed flow smolder the reaction front moves in the opposite direction as the oxidizer flow. In forward smolder, the reaction front moves in the same direction as the oxidizer flow reaching the reaction zone after passing through the hot char left by the propagating reaction while the hot postcombustion gases flow into the virgin fuel ahead of the reaction, pre-heating the fuel.

A Get Away Special Canister (GAS-CAN), carried in the payload bay of the Space Shuttle is used

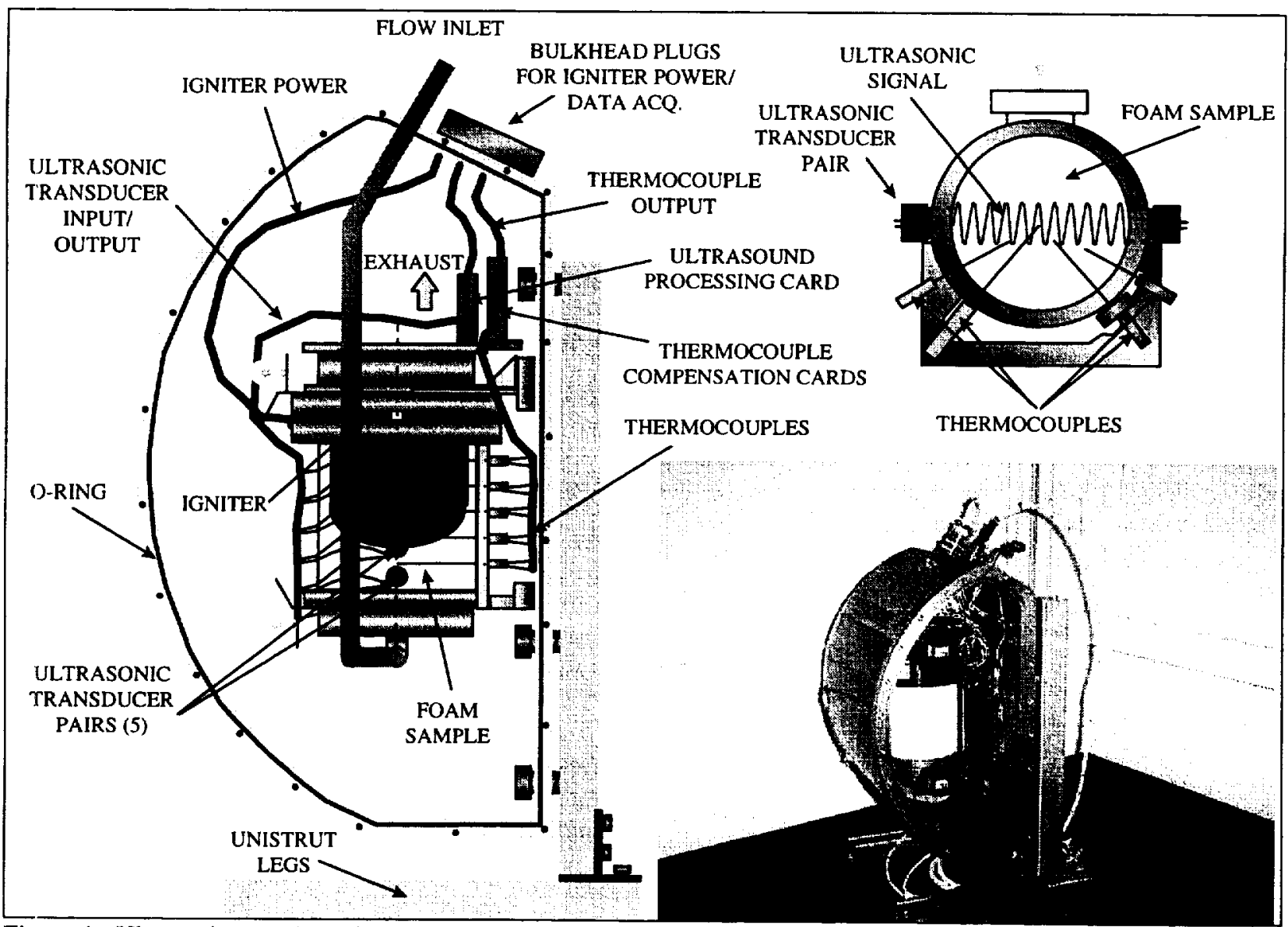

Figure 1. Ultrasonic transducer implementation into existing MSC flight hardware. Apparatus is shown in the ground-based $(1-\mathrm{g})$ opposed flow configuration. Ultrasonic transducers are not shown in photograph. 


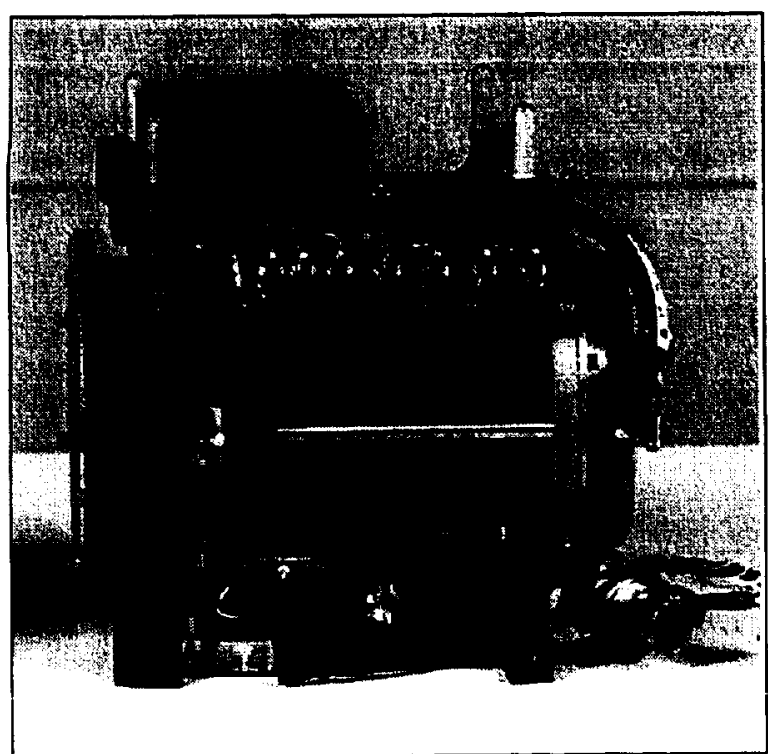

Figure 2. Photo of actual MSC test section with UIS hardware installed.

to study smolder reactions in microgravity. This apparatus is fully automated and therefore requires a well-defined ignition protocol and experimental control. Previous smoldering experiments aboard the NASA Space Shuttle (STS-69 and STS-77) initiated smoldering within the fuel sample by following a preprogrammed fuel-igniter interface, temperature-time profile. This ignition protocol was developed prior to understanding the requirements for initiating a smoldering reaction. A new protocol, developed through a series of normal-gravity tests, relies on a preset igniter power for a set time period. The power and time are set to achieve the requirements of a minimum igniter temperature and a heat flux at a particular depth $[7,8]$. This new ignition procedure has many advantages including: a reduction of the thermally assisted smolder region allowing a larger region of unassisted smolder for study, a more rigorous ignition system as there is no thermocouple feedback, and it also represents a more realistic scenario for the initiation of a smolder reaction (e.g. an overheated wire).

The ignition and propagation of smolder is further examined using an ultrasound imaging system (UIS). The UIS images provide a representation of the permeability field established within an optically opaque polyurethane foam sample in a non-intrusive fashion. The ultrasound data are compared with thermocouple histories and provide further insight into the smolder ignition process. Not only does the method allow for non-intrusive monitoring of the propagation of the smolder front, but it also allows for examination of the progress and extent of secondary char oxidation reactions occurring behind the reaction front. The UIS has been implemented into the MSC flight hardware as shown in Figure 1\&2 and will be flown aboard the remainder of the MSC Space Shuttle Missions. The technique provides information about local permeability variations within a smoldering sample, which can, in turn, be interpreted to visualize the propagation of the smolder reaction. The method utilizes the observation that transmission of an ultrasonic signal through a porous material increases with increasing permeability. (See Tse et al. [11] for in-depth discussion and analysis). Since a propagating smolder reaction leaves behind a char that is higher in permeability than the original (unburnt) material, ultrasonic transmission can be employed to monitor smolder progress. Additionally, the technique allows observation of the evolution of the char (i.e. material left by the smolder reaction), which, in certain circumstances, can continue to increase in permeability, due to secondary reactions (either oxidative or pyrolytic in nature). The UIS employed in the MSC produces a line-of-sight permeability measurement that corroborates thermocouple measurements of reaction front location and also provides information regarding the evolution of the char left in the wake of a smolder front.

\section{Experimental Set-up}

Experiments have been conducted to study the normal-gravity ignition of both forward and opposed smolder of a high void fraction, flexible, polyurethane foam in a forced oxidizer flow. The tests are performed in a $21.7 \mathrm{~L}$, semi-cylindrical, hermetically sealed, aluminum combustion chamber, identical to those used in the MSC flight assembly. The flight assembly integrates into the $0.14 \mathrm{~m}^{3}$ NASA Get Away Special Canister (GAS-CAN), located in the

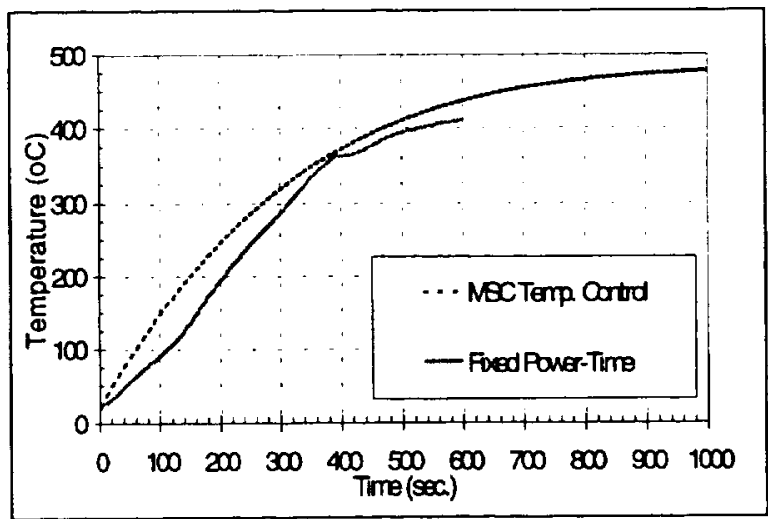

Figure 3. Igniter surface temperature profiles. The fixed power-time trace is for $1-\mathrm{g}$ downward burning. Other orientations are not shown for clarity. 
cargo bay of the Space Shuttle. The igniter consists of a Nichrome wire heater placed between two porous ceramic disks, one of which is in complete contact with the one end of the fuel sample. Gaseous oxidizer forced through the igniter and foam is controlled via mass flow controllers or choked flow nozzles. Reaction zone temperature and smolder propagation velocities are obtained from the temperature histories of thermocouples embedded at predetermined positions within the foam.

Tests have been conducted with air as oxidizer for both upward and downward orientations of both forward and opposed flow smolder $[9,10]$. Two welldefined smolder regimes are evidenced, a selfsustained smolder regime and a no-ignition regime, the boundary between which is primarily determined by two parameters, igniter power flux $\left(\mathrm{O}\left(1 \mathrm{~W} / \mathrm{cm}^{2}\right)\right)$, and the time the igniter is powered $\left(\mathrm{O}\left(10^{3} \mathrm{sec}.\right)\right)$. The required time for ignition of self-sustained smolder is shorter for forward smolder than opposed due to fuel preheating by the hot combustion products. Further, the boundary for both forward and opposed is shifted to shorter times for the same igniter power flux with increasing oxygen mass fraction $[7,8]$. An additional requirement for ignition is that the fuel-igniter interface must attain a minimum temperature. Figure 3 shows thermocouple traces for the previous ignition protocol along with the temperature trace of the new protocol. The differences in igniter surface temperature with orientation (1-g upward vs. downward - not shown) can clearly be seen using the new method, whereas the previous protocol constrained the temperature of the igniter, masking the differences. A water condensation front, the effect of reaction exothermicity, and the effects of buoyant flows all become identifiable.

The UIS system is thoroughly outlined in Tse et al. [11] and is therefore only shown here as Figure 4. The UIS has been employed in order to gain a better understanding of the smolder reaction. Briefly, operation procedures for the ultrasonic imaging technique for a single set of speaker and microphone are as follows:

1) A speaker emits an ultrasonic sinusoidal wavetrain pulse through the porous medium. The wave-train pulse consists of a given number of cycles, (6). The pulsed ultrasound signals are amplified by a gain-selectable amplifier that is controlled by a feedback loop to optimize the strength of the received signal.

2) A microphone receives the wave-form, which is amplified and converted to a RMS signal. Based on the shortest path length through the sample, the first peak in the received waveform identifies the desired transmitted signal through the foam/char.

3) The received RMS waveform strength is digitally sampled and stored.

4) The next ultrasonic transmission data point is taken. The frequency of wave-train pulses is important. The time between wave-train pulses

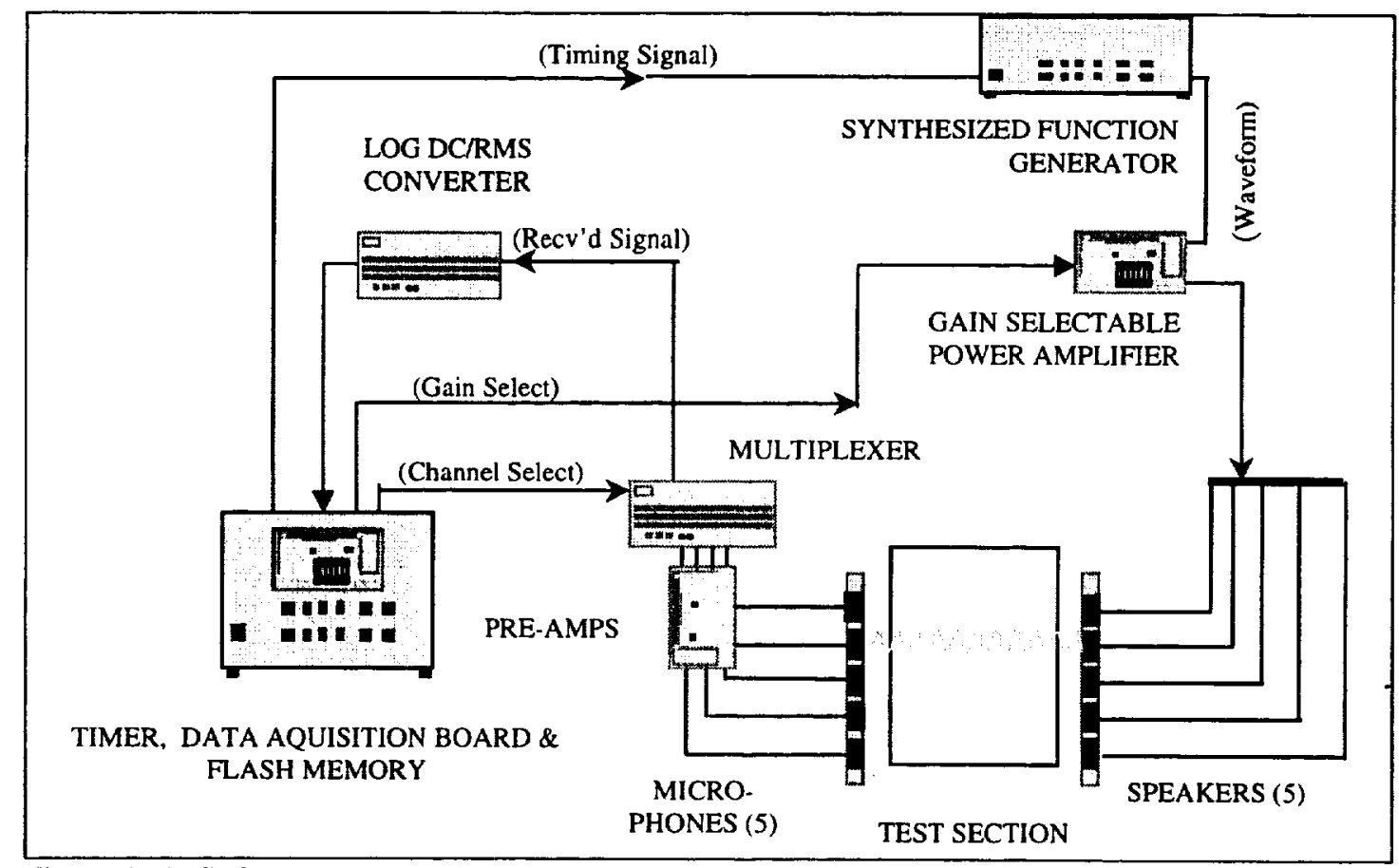

Figure 4. UIS electronic schematic 


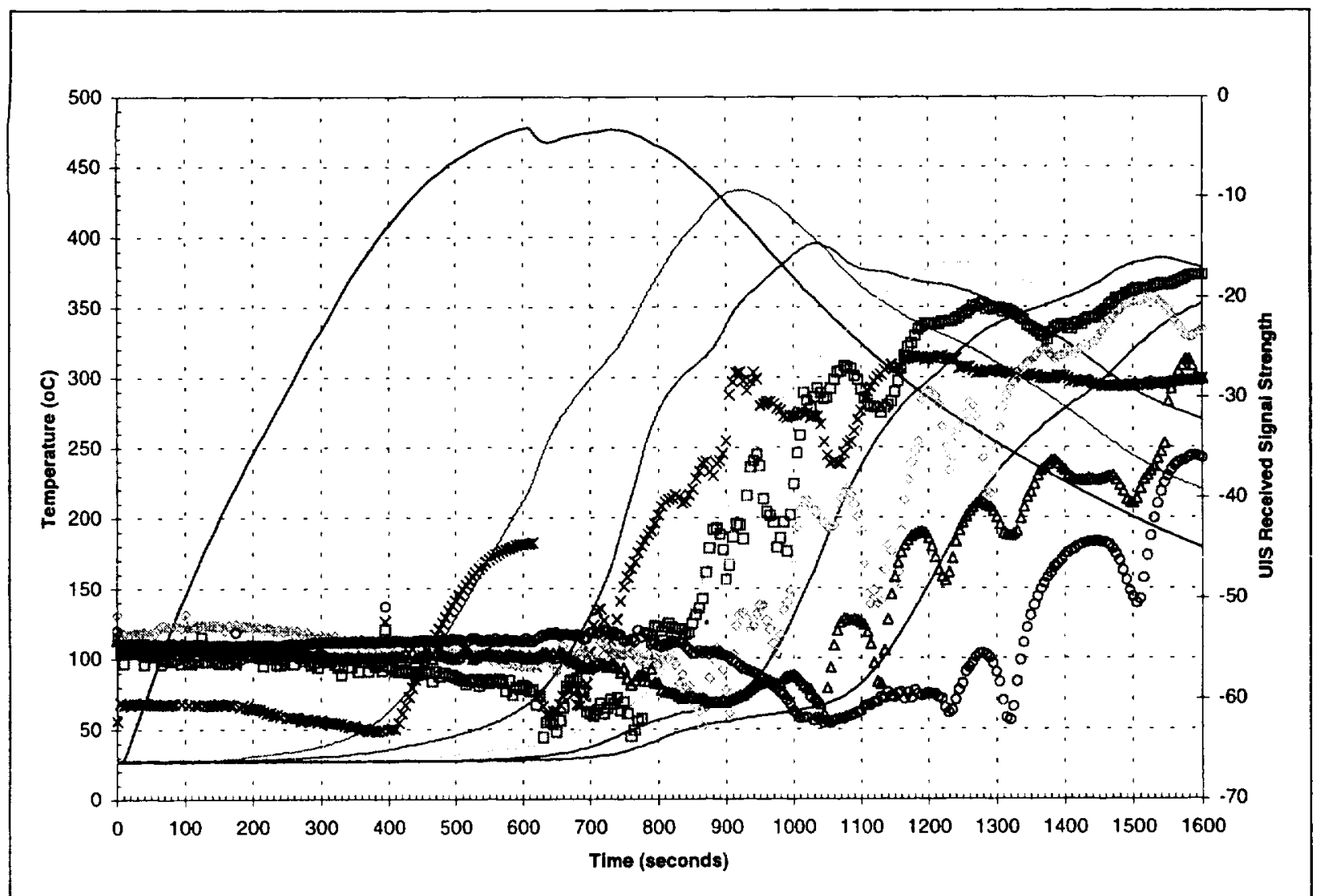

Figure 5. UIS thermocouple (solid lines) and signal (points) traces. Thermocouple positions $(0,40,60,80,100$, $120 \mathrm{~mm})$. UIS transducer pairs $(\mathrm{x}-25 \mathrm{~mm} ; \square-60 \mathrm{~mm} ; \diamond-80 \mathrm{~mm} ; \nabla-100 \mathrm{~mm} ; \mathrm{o}-120 \mathrm{~mm})$

must be longer than the time of flight for a single pulse and must also be long enough to prevent superposition effects from previously sent wavetrains reflected back into the propagation path of interest.

Five speaker/microphone pairs are fixed at 25 , $60,80,100$ and $120 \mathrm{~mm}$ from the igniter surface. This placement coincides with several thermocouples, which allows for correlation of the temperature and permeability results.

\section{Results / Discussion}

Figure 5 contains a plot of temperature and UIS received signal strength versus time. The test from which this data was taken was conducted in an upward burning, forward flow smolder with gas velocities of $0.1 \mathrm{~mm} / \mathrm{s}$ during ignition and $\approx 2.5 \mathrm{~mm} / \mathrm{s}$ during the propagation period. The UIS data shows an average smolder velocity of $0.118 \mathrm{~mm} / \mathrm{s}$. It was found from the temperature profiles that the smolder front propagated to a distance of $105 \mathrm{~mm}$ at an average velocity of $0.116 \mathrm{~mm} / \mathrm{s}$, confirming the findings of the UIS data.
It can be seen that the steep rise in transmission of the UIS signal at a given location correlates with a measured temperature of $-290-300^{\circ} \mathrm{C}$, corresponding to the pyrolysis temperature of the polyurethane foam [12]. This further corroborates the results of the UIS. The cause of the periodic nature of the UIS signal seen in Figure 5 is uncertain, however it is suspected that it may be the result of convection cells within the foam. The convection cells subtly effect the strength of reaction, causing it to produce more or less gaseous combustion products that are known to attenuate sound waves differently $[13,14]$. It is the fluctuations in gas concentration within these cells that may be attributed the received signal fluctuations. Future microgravity tests will confirm or refute these assertions.

The gradual rise and steep drop in signal transmission of Channel 1 between time $t=400-600 \mathrm{~s}$ is of interest. The sharp drop coincides with the switch from low to high oxidizer flow rate and the switching off of the igniter. This drop is caused by the passage of the water condensation front, which leads the reaction front [15]. In each channel, it may be seen from Figure 5 that the signal first undergoes a 
gradual decrease in transmission, then a sharp increase upon arrival of the actual reaction front. This gradual decrease is due to water from the reaction condensing in the cool foam ahead of the reaction. The water partially fills the pores of the foam, increasing the attenuation of the ultrasound signal. With the arrival of the reaction front, the water is evaporated, and the permeability increases, increasing the transmission of the UIS signal. This is seen in every channel and is corroborated by the leveling off of the thermocouple traces at $\sim 75^{\circ} \mathrm{C}$ (most notably TC's 4-6), an indication of an endothermic event such as water condensation. Peculiar to Channel 1 , however is the sharp drop in signal after a short time of increasing signal. This is due to the large amount of preheated, unreacted fuel, generated by the low oxidizer flow during the ignition phase of the test. When the oxidizer flow is switched to the higher rate, this fuel reacts quickly, generating large amounts of products, which rapidly fill the pores ahead of the reaction. This results in the sharp drop in signal transmission evidenced in Figure 5. Also coincident with the oxidizer flow rate switch, Channel 2 undergoes a sharp drop in signal transmission, although not as severe.

\section{Concluding Remarks}

An ultrasound imaging system has been developed and implemented into the ongoing Microgravity Smoldering Combustion experiments. The data collected will provide further insight into the smoldering process and provide a non-intrusive method for studying a smolder reaction propagating through a permeable material. The method has successfully imaged both the reaction front and the preceding water condensation front. Further, a new ignition procedure has been implemented which more accurately simulates a smolder ignition hazard and allows for a larger portion of the sample to under-go self-supporting smolder.

\section{Acknowledgments}

This work was supported by the National Aeronautics and Space Administration under NASA grants NAG3-1252 and NAG3-2026. The authors would like to acknowledge the work and support of Ms. S. Motil and the NASA Lewis engineering team.

\section{$\underline{\text { References }}$}

1. Ohlemiller, T.J., Prog. Ener. \& Comb. Sci., 11:277, (1986).
2. Ortiz-Molina, M.G., Toong, T.Y., Moussa, N.A., and Tesero, G.C., Seventeenth Symposium (International) on Combustion, The Combustion Institute, pp.1191, (1979).

3. Damant, G.H., J. Textile. Inst., 85:505, (1994).

4. Ross, H.D., "Invited Lecture," Natl. Fire Protection Assoc. Annual Meeting, May, 1996.

5. Friedman, R., NASA Technical Memorandum $106403,1994$.

6. Palmer, H., "Closing Address," International Microgravity Workshop, NASA LeRC, Cleveland, OH, January 25, 1989.

7. Walther, D.C., Anthenien, R.A., and FernandezPello, A.C., "The effect of oxygen concentration on the ignition of smolder of polyurethane foam", Mediterranean Combustion Symposium, 1999 (submitted).

8. Anthenien, R.A. and Fernandez-Pello, A.C. Twenty-seventh Symposium (International) on Combustion, The Combustion Institute (in press).

9. Anthenien, R.A. "A Study of Ignition of Forward Smoldering of Polyurethane Foam," PhD Thesis, Berkeley, CA (1998).

10. Walther, D.C. "A Study of Opposed Flow Smoldering of Polyurethane Foam: Comparison between Normal- and Microgravity," Ph.D. Thesis, Berkeley, CA (1998).

11. Tse, S.D., Anthenien, R.A., Fernandez-Pello A.C., and Miyasaka, K., Comb \& Flame 116:120 (1999).

12. Bilbao, R., Mastral, J.F., Ceamanos, J., and Aldea, M.F., J. Macromolec. Sci. Chem.

A15(1):169 (1996).

13. Herzfeld, K., and Litovitz, T., Absorption of Ultrasonic Waves, Academic Press, Inc., New York (1959).

14. Bhatia, A., Ultrasonic Absorption, Oxford University Press, New York (1967).

15. DeRis, J., Comb. Sci. Tech. 2:239 (1970). 